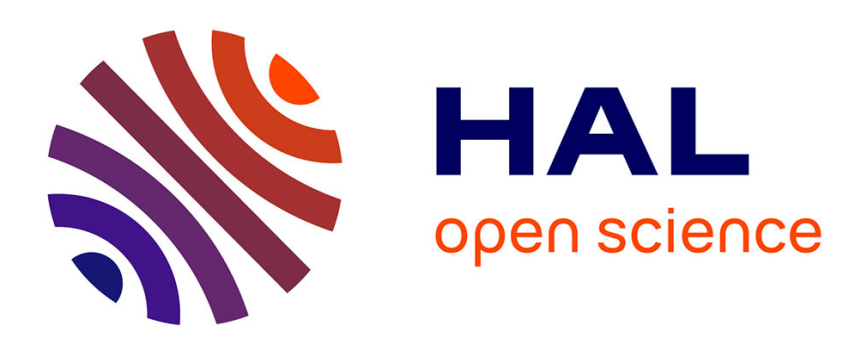

\title{
Tables et diagrammes universels des résonances de cavités coaxiales ou concentriques
}

A. Poinsot

\section{To cite this version:}

A. Poinsot. Tables et diagrammes universels des résonances de cavités coaxiales ou concentriques. Revue de Physique Appliquée, 1979, 14 (2), pp.445-450. 10.1051/rphysap:01979001402044500 . jpa00244613

\section{HAL Id: jpa-00244613 https://hal.science/jpa-00244613}

Submitted on 1 Jan 1979

HAL is a multi-disciplinary open access archive for the deposit and dissemination of scientific research documents, whether they are published or not. The documents may come from teaching and research institutions in France or abroad, or from public or private research centers.
L'archive ouverte pluridisciplinaire HAL, est destinée au dépôt et à la diffusion de documents scientifiques de niveau recherche, publiés ou non, émanant des établissements d'enseignement et de recherche français ou étrangers, des laboratoires publics ou privés. 


\title{
Tables et diagrammes universels des résonances de cavités coaxiales ou concentriques
}

\author{
A. Poinsot \\ Laboratoire de Spectronomie $(*)$, groupe de Radioélectricité, Université de Dijon, \\ 6, bd Gabriel, 21000 Dijon, France
}

(Reçu le 28 juillet 1978, révisé le 18 octobre 1978, accepté le 20 octobre 1978)

\begin{abstract}
Résumé. - Cet article donne des tables universelles permettant de calculer les résonances d'une cavité électromagnétique délimitée par deux cylindres coaxiaux ou deux sphères concentriques, pour toute valeur comprise entre 0 et 1 du rapport $R_{1} / R_{2}$. Les modes de résonance se classent en deux familles qui se distinguent par leurs comportements asymptotiques quand $R_{1} / R_{2} \rightarrow 1$.
\end{abstract}

\begin{abstract}
This paper gives universal tables for the calculation of natural frequencies in an electromagnetic cavity delimited by two coaxial circular cylinders or two concentric spheres, for any value of the ratio $R_{1} / R_{2}$ between 0 and 1 . Modes of resonance fall in two families which can be distinguished by their asymptotic properties when $R_{1} / R_{2} \rightarrow 1$.
\end{abstract}

1. Cavité coaxiale. - Une cavité coaxiale de paramètres géométriques $R_{1}, R_{2}, L$ (Fig. 1) possède une infinité de fréquences de résonance définies par des équations implicites résultant de l'écriture des conditions aux limites [1].

Posons

$K=\left[\left(\frac{2 \pi v}{c}\right)^{2}-\left(\frac{n \pi}{L}\right)^{2}\right]^{1 / 2}$.
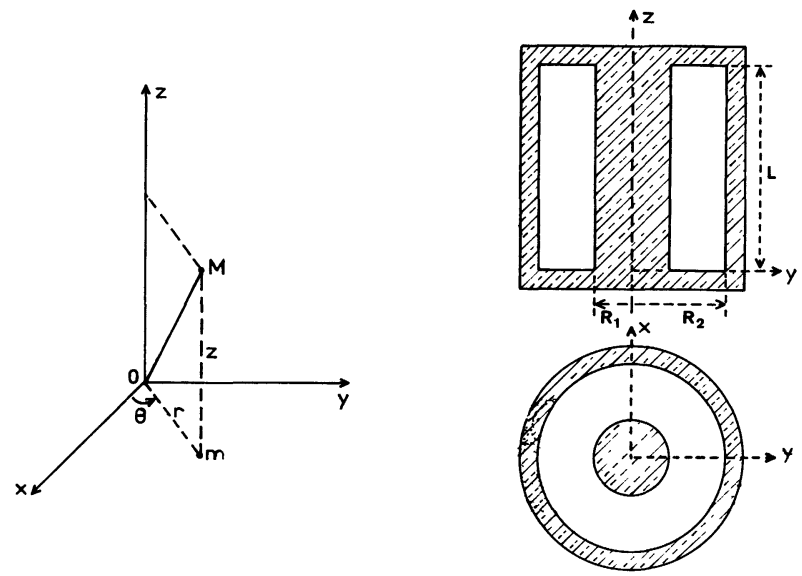

Fig. 1. - Paramètres de définition de la cavité coaxiale.

[Parameters defining the coaxial type cavity.]

$\left(^{*}\right)$ E.R.A. $n^{\circ} 599$.
Les fréquences de résonance $v$ sont alors les solutions des équations caractéristiques suivantes :

$$
\text { Modes } \text { TEM }_{00 n}
$$

$K=0$

Modes $\mathrm{TM}_{l m n}$

$J_{l}\left(K R_{1}\right) Y_{l}\left(K R_{2}\right)-J_{l}\left(K R_{2}\right) Y_{l}\left(K R_{1}\right)=0$.

Modes $\mathrm{Te}_{l m n}$

$J_{l}^{\prime}\left(K R_{1}\right) Y_{l}^{\prime}\left(K R_{2}\right)-J_{l}^{\prime}\left(K R_{2}\right) Y_{l}^{\prime}\left(K R_{1}\right)=0$.

$J_{l}$ et $Y_{l}$ sont les fonctions de Bessel de première et seconde espèces.

$J_{l}^{\prime}$ et $Y_{l}^{\prime}$ sont les fonctions dérivées.

La désignation d'un mode de résonance fait appel à 3 indices, $l, m, n$ liés à la topographie du champ électromagnétique dans le résonateur. Deux indices seulement $l$ et $n$ interviennent explicitement dans les équations caractéristiques. Le troisième indice, $m$, donne le rang de la solution considérée, dans la suite infinie des solutions classées en ordre croissant.

L'indice $l$ est lié à la variation azimutale du champ, $2 l$ étant le nombre de maxima rencontrés le long d'une circonférence où $\theta$ seul varie. La plus petite valeur permise, $l=0$, correspond à une topographie du champ possédant une symétrie de révolution.

L'indice $m$ est lié à la répartition radiale du champ et donne le nombre des maxima de champ électrique 
rencontrés le long d'un rayon de la cavité. La plus petite valeur permise est $m=1$ (sauf pour le mode TEM).

Enfin l'indice $n$ est lié à la répartition axiale du champ et donne le nombre des maxima de champ électrique rencontrés le long d'une direction parallèle à l'axe de la cavité. La plus petite valeur permise est $n=0$ pour les modes TM et $n=1$ pour les modes TE. Lorsque $n=0$ le champ ne dépend pas de la coordonnée $z$.

Les solutions des équations (3) et (4) ont déjà été tabulées en fonction du paramètre $R_{2} / R_{1}$ qui peut varier de 1 à $+\infty$. De telles tables [2] sont nécessairement tronquées et il nous a semblé préférable d'établir des tables donnant les solutions en fonction du paramètre $R_{1} / R_{2}$ variant de 0 à 1 .

Ces tables donnent les valeurs de $K\left(R_{2}-R_{1}\right)$ qui satisfont aux équations (2) et (3) pour une valeur donnée du rapport $R_{1} / R_{2}$. Les valeurs de $K\left(R_{2}-R_{1}\right)$ se situent toujours dans un domaine borné, inférieurement et supérieurement. Les bornes obtenues pour $R_{1}=0$ donnent les fréquences de résonance d'une cavité cylindrique de rayon $R_{2}$. Ce sont donc les racines des fonctions de Bessel $J_{l}$ (modes TM) ou de leurs dérivées $J_{l}^{\prime}$ (modes TE). Les bornes obtenues pour $R_{1}=R_{2}$ sont des multiples du nombre $\pi$.

Les modes ont été classés selon leurs propriétés asymptotiques quand $R_{1} / R_{2} \rightarrow 1$, puis, à l'intérieur des familles ainsi définies, par leurs propriétés asymptotiques quand $R_{1} / R_{2} \rightarrow 0$.
Cette façon de procéder met en évidence le comportement particulier des modes $\mathrm{TE}_{0 m n}$ qui se trouvent classés à la fin de la famille $\mathrm{TE}_{l m n}$.

Pour utiliser ces tables il convient de se fixer un mode dont la topographie du champ électromagnétique convienne à l'application envisagée. $R_{1}$ et $R_{2}$ sont fixés arbitrairement. Les tables permettent, à l'aide de la relation (1), de déterminer la hauteur $L$ de la cavité si l'on se fixe la fréquence de résonance $v$, ou inversement de déterminer $v$ dans une cavité de hauteur donnée.

On peut aussi présenter les résultats sous forme graphique en portant $K R_{2}$ en fonction de $R_{1} / R_{2}$. La figure 2 montre le diagramme obtenu pour les 20 premiers modes du résonateur coaxial. Ce diagramme appelle quelques commentaires.

- Les modes ayant mêmes indices $l$ et $m$, mais des indices $n$ différents sont confondus sur ce diagramme, mais cela ne signifie pas qu'ils résonnent à la même fréquence, car la constante $K$ dépend de $n$. En fait les dégénérescences que l'on observe dans le résonateur coaxial sont les mêmes que dans la cavité cylindrique. Les modes $\mathrm{TE}_{0 m n}$ sont dégénérés avec les modes $\mathrm{TM}_{1 m n}$ du fait que $J_{0}^{\prime}=-J_{1}$ et $Y_{0}^{\prime}=-Y_{1}$.

- La fréquence de résonance croît en même temps que $R_{1} / R_{2}$, sauf dans le cas des modes $\mathrm{TE}_{l 1 n}$ avec $l \neq 0$.

- Lorsque $R_{1} \rightarrow R_{2}$ les modes se regroupent et adoptent un comportement asymptotique commun

Résonateur coaxial - Modes $\mathrm{TM}_{l m n}$ : valeurs de $K\left(R_{2}-R_{1}\right)$ satisfaisant l'équation $J_{l}\left(K R_{1}\right) Y_{l}\left(K R_{2}\right)-J_{l}\left(K R_{2}\right) Y_{l}\left(K R_{1}\right)=0$.

\begin{tabular}{|c|c|c|c|c|c|c|c|c|c|}
\hline \multirow[b]{2}{*}{$R_{1} / R_{2}$} & \multicolumn{9}{|c|}{$l, m$} \\
\hline & 0,1 & 1,1 & 2,1 & 3,1 & 4,1 & 0,2 & 1,2 & 2,2 & 0,3 \\
\hline$\overline{0}$ & $2, \overline{405}$ & $3 \overline{832}$ & $5 \overline{136}$ & $6 \overline{380}$ & $7 \overline{588}$ & $5, \overline{520}$ & $\overline{7,016}$ & $8 \overline{417}$ & $8, \overline{654}$ \\
\hline 0,05 & 2,911 & 3,667 & 4,879 & 6,061 & 7,209 & 6,104 & 6,749 & 7,999 & 9,278 \\
\hline 0,10 & 2,983 & 3,547 & 4,628 & 5,742 & 6,830 & 6,172 & 6,597 & 7,612 & 9,340 \\
\hline 0,15 & 3,024 & 3,457 & 4,391 & 5,426 & 6,450 & 6,208 & 6,505 & 7,290 & 9,369 \\
\hline 0,20 & 3,053 & 3,389 & 4,177 & 5,116 & 6,072 & 6,228 & 6,444 & 7,043 & 9,386 \\
\hline 0,25 & 3,073 & 3,336 & 3,990 & 4,820 & 5,699 & 6,243 & 6,403 & 6,858 & 9,396 \\
\hline 0,30 & 3,089 & 3,294 & 2,829 & 4,546 & 5,336 & 6,253 & 6,373 & 6,720 & 9,404 \\
\hline 0,35 & 3,101 & 3,261 & 3,693 & 4,298 & 4,992 & 6,260 & 6,351 & 6,616 & 9,409 \\
\hline 0,40 & 3,110 & 3,235 & 3,580 & 4,080 & 4,674 & 6,266 & 6,335 & 6,537 & 9,413 \\
\hline 0,45 & 3,117 & 3,214 & 3,485 & 3,890 & 4,387 & 6,270 & 6,322 & 6,475 & 9,416 \\
\hline 0,50 & 3,123 & 3,197 & 3,407 & 3,729 & 4,133 & 6,273 & 6,312 & 6,428 & 9,418 \\
\hline 0,55 & 3,128 & 3,183 & 3,343 & 3,593 & 3,914 & 6,276 & 6,305 & 6,390 & 9,420 \\
\hline 0,60 & 3,131 & 3,172 & 3,291 & 3,479 & 3,727 & 6,278 & 6,299 . & 6,361 & 9,421 \\
\hline 0,65 & 3,134 & 3,163 & 3,249 & 3,387 & 3,571 & 6,279 & 6,294 & 6,339 & 9,422 \\
\hline 0,70 & 3,137 & 3,157 & 3,216 & 3,312 & 3,443 & 6,281 & 6,291 & 6,321 & 9,423 \\
\hline 0,75 & 3,138 & 3,151 & 3,190 & 3,254 & 3,341 & 6,281 & 6,288 & 6,310 & 9,424 \\
\hline 0,80 & 3,140 & 3,147 & 3,171 & 3,210 & 3,264 & 6,282 & 6,286 & 6,298 & 9,424 \\
\hline 0,85 & 3,141 & 3,145 & 3,157 & 3,178 & 3,207 & 6,283 & 6,285 & 6,291 & 9,424 \\
\hline 0,90 & 3,141 & 3,143 & 3,148 & 3,157 & 3,169 & 6,283 & 6,284 & 6,286 & 9,425 \\
\hline 0,95 & 3,142 & 3,142 & 3,143 & 3,145 & 3,148 & 6,283 & 6,283 & 6,284 & 9,425 \\
\hline 1 & 3,142 & 3,142 & 3,142 & 3,142 & 3,142 & 6,283 & 6,283 & 6,283 & 9,425 \\
\hline
\end{tabular}


Résonateur coaxial - Modes $\mathrm{TE}_{l m n}$ : valeurs de $K\left(R_{2}-R_{1}\right)$ satisfaisant l'équation $J_{l}^{\prime}\left(K R_{1}\right) Y_{l}^{\prime}\left(K R_{2}\right)-J_{l}^{\prime}\left(K R_{2}\right) Y_{l}^{\prime}\left(K R_{1}\right)=0$.

\begin{tabular}{|c|c|c|c|c|c|c|c|c|c|}
\hline \multirow[b]{2}{*}{$R_{1} / R_{2}$} & \multicolumn{9}{|c|}{$l, m$} \\
\hline & 1,1 & 2,1 & 3,1 & 4,1 & 0,1 & 1,2 & 2,2 & 0,2 & 1,3 \\
\hline 0 & 1,841 & 3,054 & 4,201 & 5,317 & 3,832 & 5,331 & 6,706 & 7,016 & 8,536 \\
\hline 0,05 & 1,740 & 2,901 & 3,991 & 5,032 & 3,667 & 5,011 & 6,372 & 6,749 & 7,982 \\
\hline 0,10 & 1,623 & 2,748 & 3,781 & 4,786 & 3,547 & 4,623 & 6,018 & 6,597 & 7,379 \\
\hline 0,15 & 1,496 & 2,591 & 3,571 & 4,520 & 3,457 & 4,259 & 5,627 & 6,505 & 6,979 \\
\hline 0,20 & 1,364 & 2,428 & 3,359 & 4,254 & 3,389 & 3,969 & 5,196 & 6,444 & 6,746 \\
\hline 0,25 & 1,233 & 2,257 & 3,145 & 3,987 & 3,336 & 3,753 & 4,768 & 6,403 & 6,606 \\
\hline 0,30 & 1,107 & 2,078 & 2,926 & 3,719 & 3,294 & 3,596 & 4,391 & 6,373 & 6,515 \\
\hline 0,35 & 0,988 & 1,893 & 2,700 & 3,447 & 3,261 & 3,481 & 4,087 & 6,351 & 6,454 \\
\hline 0,40 & 0,877 & 1,705 & 2,465 & 3,169 & 3,235 & 3,395 & 3,850 & 6,335 & 6,410 \\
\hline 0,45 & 0,773 & 1,520 & 2,223 & 2,883 & 3,214 & 3,331 & 3,669 & 6,322 & 6,378 \\
\hline 0,50 & 0,677 & 1,341 & 1,979 & 2,588 & 3,197 & 3,282 & 3,531 & 6,312 & 6,353 \\
\hline 0,55 & 0,588 & 1,169 & 1,737 & 2,287 & 3,183 & 3,245 & 3,427 & 6,305 & 6,335 \\
\hline 0,60 & 0,505 & 1,006 & 1,502 & 1,988 & 3,172 & 3,216 & 3,347 & 6,299 & 6,320 \\
\hline 0,65 & 0,426 & 0,853 & 1,276 & 1,695 & 3,163 & 3,194 & 3,286 & 6,294 & 6,309 \\
\hline 0,70 & 0,355 & 0,709 & 1,062 & 1,413 & 3,157 & 3,178 & 3,240 & 6,291 & 6,301 \\
\hline 0,75 & 0,287 & 0,573 & 0,859 & 1,145 & 3,151 & 3,165 & 3,205 & 6,288 & 6,295 \\
\hline 0,80 & 0,223 & 0,445 & 0,668 & 0,890 & 3,147 & 3,156 & 3,179 & 6,286 & 6,290 \\
\hline 0,85 & 0,162 & 0,325 & 0,487 & 0,649 & 3,145 & 3,149 & 3,162 & 6,285 & 6,287 \\
\hline 0,90 & 0,105 & 0,211 & 0,316 & 0,421 & 3,143 & 3,145 & 3,150 & 6,284 & 6,285 \\
\hline 0,95 & 0,051 & 0,103 & 0,154 & 0,205 & 3,142 & 3,142 & 3,144 & 6,283 & 6,284 \\
\hline 1 & 0 & 0 & 0 & 0 & 3,142 & 3,142 & 3,142 & 6,283 & 6,283 \\
\hline
\end{tabular}

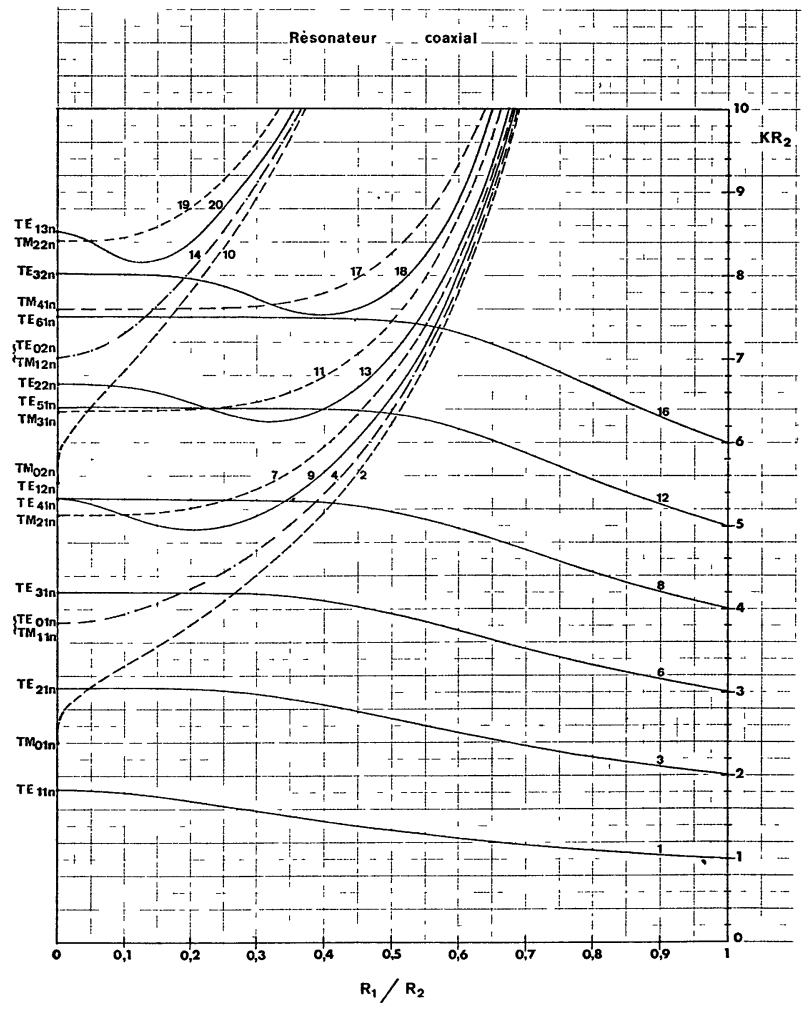

Fig. 2. - Diagramme universel de résonance pour les 20 premiers modes de la cavité coaxiale (modes $\mathrm{TE}_{l m n}$ en traits pleins et modes $\mathrm{TM}_{l m n}$ en tiretés).

[Universal diagram for the 20 lowest resonant modes in the coaxial type cavity (continuous lines for $\mathrm{TE}_{l m n}$ modes and broken lines for the $\mathrm{TM}_{l m n}$ modes).] qui peut être calculé facilement en considérant la cavité coaxiale comme un guide rectangulaire de section $\left(R_{2}-R_{1}\right) \times L$, refermé sur lui-même. En écrivant que la longueur de la circonférence moyenne est un multiple de la longueur d'onde dans le guide soit

$\pi\left(R_{1}+R_{2}\right)=l \lambda_{\mathrm{g}}$

et en introduisant la constante $K$ définie par (1) on obtient

$\left[K R_{2}\right]^{2}=\left[\frac{2 l}{1+R_{1} / R_{2}}\right]^{2}+\left[\frac{m \pi}{1-R_{1} / R_{2}}\right]^{2}$.

Dans cette expression, le premier terme décrit le comportement asymptotique des modes $\mathrm{TE}_{l 1 n}$ dont la fréquence est une fonction décroissante de $R_{1} / R_{2}$, tandis que le second terme donne le comportement asymptotique des autres modes, dont la fréquence croît avec $R_{1} / R_{2}$. Les figures 3 et 4 permettent de comparer les solutions exactes avec l'approximation du guide rectangulaire.

- Notons enfin une particularité des modes $\mathbf{T M}_{0 m n}$ qui sont les seuls à aborder l'axe des ordonnées avec une tangente verticale quand $R_{1} / R_{2} \rightarrow 0$.

2. Cavité concentrique. - La cavité formée par deux sphères concentriques, supposées parfaitement conductrices, présente également une infinité de réso- 


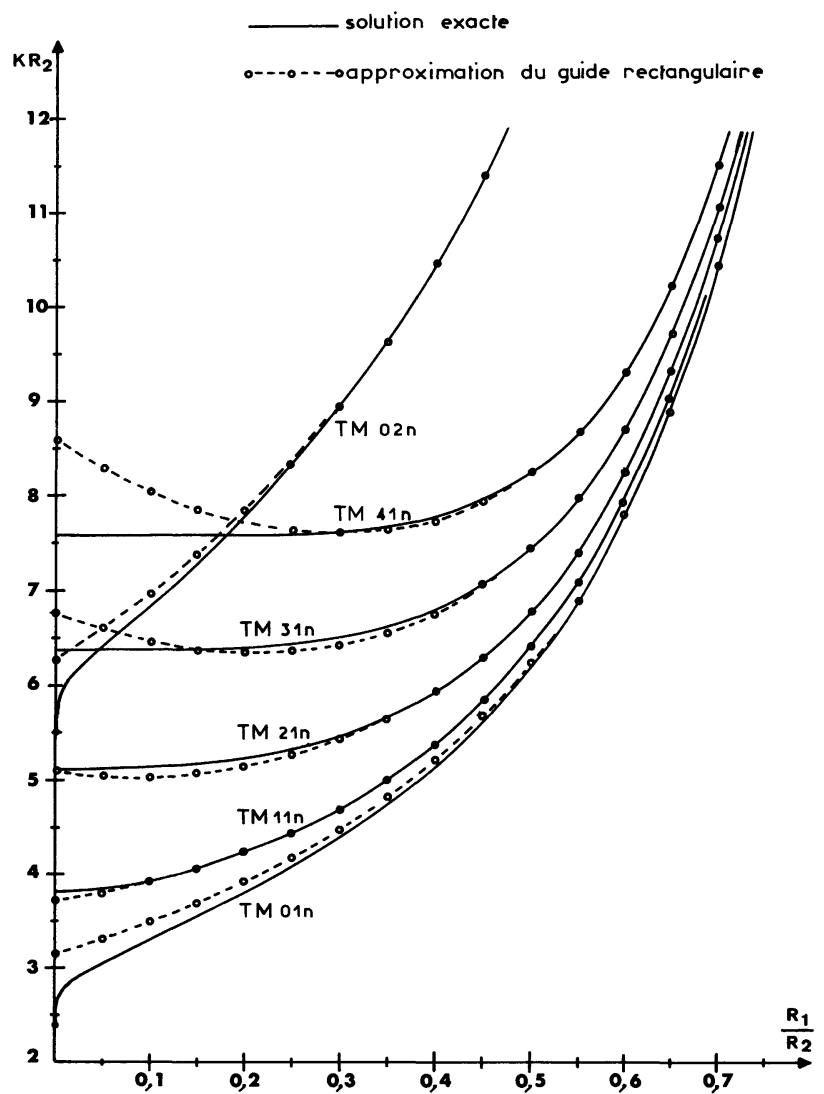

Fig. 3. - Solution exacte (traits pleins) et approximation du guide rectangulaire (tirets) pour les modes $\mathrm{TM}_{l m n}$ du résonateur coaxial.

[Exact solution (solid curves) and approximation of a rectangular waveguide (dashed curves) for $\mathrm{TM}_{l_{m n}}$ modes in the coaxial-type resonator.]

nances dont les fréquences sont les solutions des équations caractéristiques suivantes, où $k=2 \pi v / c$ :

Modes $\mathbf{T M}_{n m p}$

$j_{n}^{*}\left(k R_{1}\right) y_{n}^{*}\left(k R_{2}\right)-j_{n}^{*}\left(k R_{2}\right) y_{n}^{*}\left(k R_{1}\right)=0$.

Modes $\mathrm{TE}_{n m p}$

$j_{n}\left(k R_{1}\right) y_{n}\left(k R_{2}\right)-j_{n}\left(k R_{2}\right) y_{n}\left(k R_{1}\right)=0$

$j_{n}$ et $y_{n}$ sont les fonctions de Bessel sphériques de première et seconde espèces. On a ici

$j_{n}^{*}(x)=\frac{\mathrm{d}}{\mathrm{d} x}\left[x j_{n}(x)\right]$.

$y_{n}^{*}(x)$ est défini à partir de $y_{n}(x)$ par une relation analogue.

Ici encore, trois indices $n, m, p$, sont nécessaires pour désigner une résonance, c'est-à-dire une topographie du champ électromagnétique compatible avec les parois métalliques.

L'indice $n=1,2, \ldots, \infty$ est lié à la répartition du champ selon la colatitude $\theta$ (Fig. 5) par la fonction $P_{n}^{m}(\cos \theta)$ et sa dérivée.

L'indice $m=0,1, \ldots, n$ est lié à la répartition du champ selon la longitude $\varphi$ par les fonctions $\sin m \varphi$ et $\cos m \varphi$.

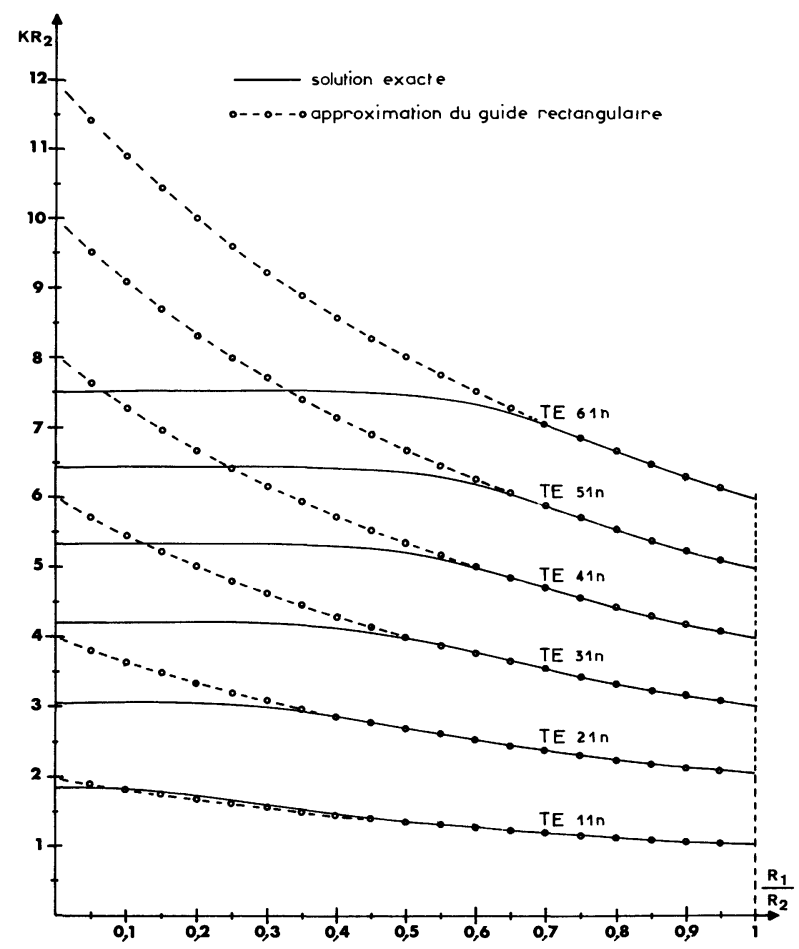

Fig. 4. - Solution exacte (traits pleins) et approximation du guide rectangulaire (tirets) pour les modes $\mathrm{TE}_{l 1 n}$ du résonateur coaxial.

[Exact solution (solid curves) and approximation of a rectangular waveguide (dashed curves) for $\mathrm{TE}_{l 1 n}$ modes in the coaxial type resonator.]
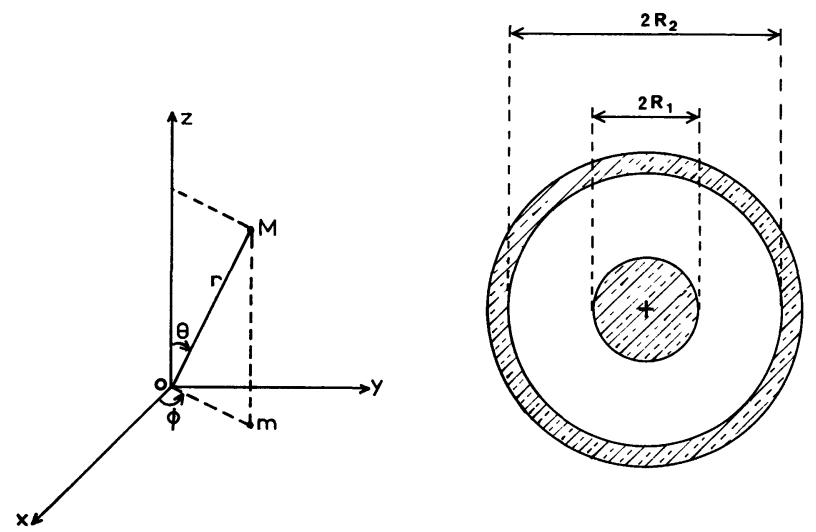

Fig. 5. - Paramètres de définition de la cavité concentrique.

[Parameters defining the concentric type cavity.]

L'indice $p=1,2, \ldots, \infty$ est lié à la répartition radiale du champ par les fonctions $j_{n}(k r), y_{n}(k r)$, $j_{n}^{*}(k r)$ et $y_{n}^{*}(k r)$.

Seul l'indice $n$ intervient explicitement dans les équations caractéristiques. L'indice $p$ donne le rang de la solution considérée, dans la suite infinie des solutions classées en ordre croissant. Les modes ayant mêmes indices $n$ et $p$, mais des indices $m$ différents résonnent à la même fréquence, bien que les topographies du champ électromagnétique soient différentes. On trouve donc de nombreuses dégénérescences, identiques d'ailleurs à celles que l'on rencontre dans la cavité sphérique. 


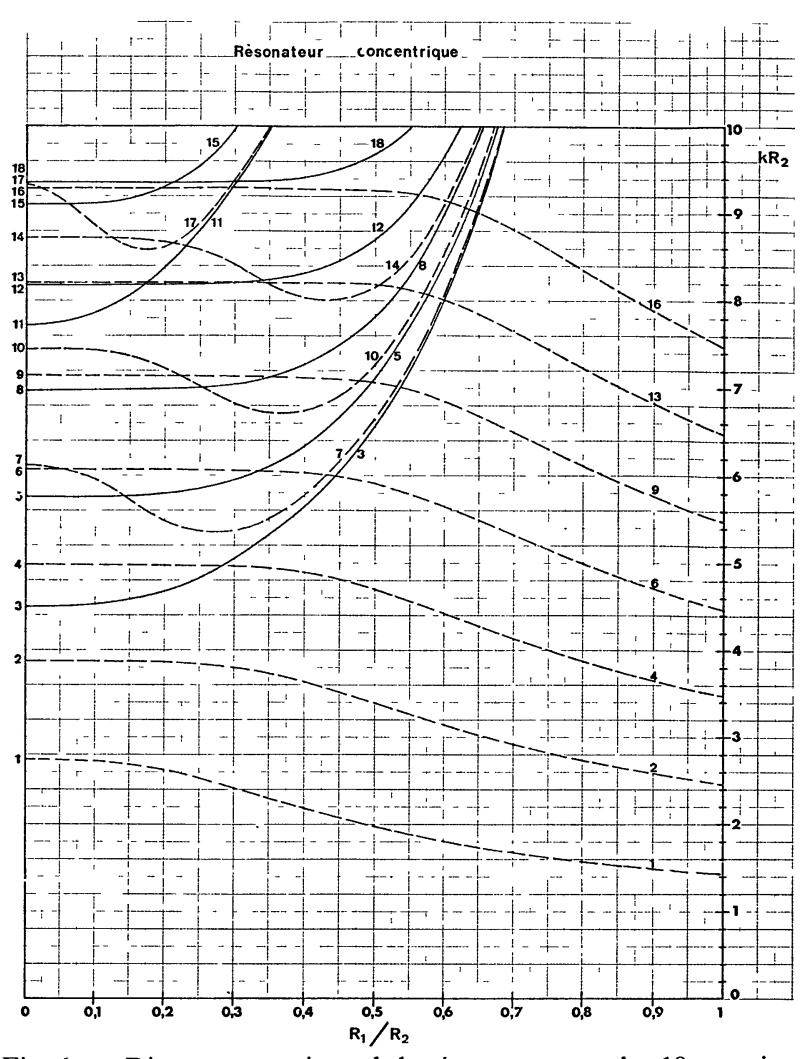

Fig. 6. - Diagramme universel de résonance pour les 18 premiers modes de la cavité concentrique (modes $\mathrm{TE}_{n m p}$ en traits pleins et modes $\mathrm{TM}_{n m p}$ en tiretés).

[Universal diagram for the 18 lowest resonant modes in the concentric type cavity (continuous lines for $\mathrm{TE}_{n m p}$ modes and broken lines for $\mathrm{TM}_{n m p}$ modes).]
A notre connaissance les solutions de (6) et (7) n'ont pas été tabulées. Nous présentons donc deux tables donnant les valeurs de $k\left(R_{2}-R_{1}\right)$ qui satisfont les équations (6) et (7) pour une valeur donnée du rapport $R_{1} / R_{2}$. Ces valeurs de $k\left(R_{2}-R_{1}\right)$ restent, pour chaque mode, dans un intervalle borné inférieurement et supérieurement. Les bornes obtenues pour $R_{1}=0$ donnent les résonances d'une cavité sphérique. Ce sont donc les racines des fonctions de Bessel sphériques $j_{n}$ (modes TM) ou $j_{n}^{*}$ (modes TE). Les bornes obtenues pour $R_{1}=R_{2}$ sont ici encore les multiples du nombre $\pi$.

La figure 6 montre le graphique obtenu en portant $k R_{2}$ en fonction de $R_{1} / R_{2}$.

On y trouve une famille de modes qui se distinguent des autres par le fait que leur fréquence de résonance est une fonction décroissante de $R_{1} / R_{2}$. Ce sont ici les modes $\mathrm{TM}_{n m 1}$.

Pour tous les autres modes la fréquence croît avec $R_{1} / R_{2}$ et on retrouve, comme dans la cavité coaxiale, un comportement asymptotique donné par

$k\left(R_{2}-R_{1}\right) \rightarrow m \pi$

quand $R_{1} \rightarrow R_{2}$.

A la différence du résonateur coaxial, quand $R_{1} \rightarrow 0$, tous les modes du résonateur concentrique abordent l'axe des ordonnées avec une tangente horizontale.

On remarquera que tous les résultats présentés ici, restent valables dans le cas où la cavité est complète-

Résonateur concentrique - Modes $\mathrm{TM}_{n m p}$ : valeurs de $k\left(R_{2}-R_{1}\right)$ satisfaisant l'équation

$j_{n}^{*}\left(k R_{1}\right) 1_{n}^{*}\left(k R_{2}\right)-j_{n}^{*}\left(k R_{2}\right) l_{n}^{*}\left(k R_{1}\right)=0$.

$\begin{array}{llllllllll}R_{1} / R_{2} & 1,1 & 2,1 & 3,1 & 4,1 & 5,1 & 1,2 & 2,2 & 3,2 & 1,3 \\ - & - & - & - & - & - & - & - & - & - \\ 0 & 2,744 & 3,870 & 4,973 & 6,062 & 7,140 & 6,117 & 7,443 & 8,720 & 9,317 \\ 0,05 & 2,605 & 3,677 & 4,725 & 5,759 & 6,783 & 5,792 & 7,071 & 8,286 & 8,784 \\ 0,10 & 2,454 & 3,483 & 4,476 & 5,456 & 6,426 & 5,366 & 6,692 & 7,849 & 7,977 \\ 0,15 & 2,285 & 3,287 & 4,227 & 5,153 & 6,069 & 4,840 & 6,282 & 7,409 & 7,270 \\ 0,20 & 2,096 & 3,087 & 3,978 & 4,850 & 5,712 & 4,356 & 5,805 & 6,952 & 6,886 \\ 0,25 & 1,893 & 2,877 & 3,726 & 4,546 & 5,355 & 3,994 & 5,277 & 6,450 & 6,682 \\ 0,30 & 1,689 & 2,655 & 3,470 & 4,241 & 4,998 & 3,745 & 4,776 & 5,892 & 6,562 \\ 0,35 & 1,493 & 2,420 & 3,206 & 3,933 & 4,639 & 3,574 & 4,362 & 5,327 & 6,485 \\ 0,40 & 1,310 & 2,175 & 2,929 & 3,618 & 4,278 & 3,456 & 4,044 & 4,820 & 6,431 \\ 0,45 & 1,144 & 1,931 & 2,640 & 3,292 & 3,910 & 3,371 & 3,806 & 4,405 & 6,393 \\ 0,50 & 0,992 & 1,693 & 2,344 & 2,954 & 3,531 & 3,309 & 3,630 & 4,080 & 6,364 \\ 0,55 & 0,854 & 1,467 & 2,050 & 2,607 & 3,139 & 3,263 & 3,498 & 3,832 & 6,342 \\ 0,60 & 0,729 & 1,256 & 1,765 & 2,259 & 2,738 & 3,229 & 3,398 & 3,641 & 6,326 \\ 0,65 & 0,613 & 1,060 & 1,494 & 1,920 & 2,338 & 3,203 & 3,322 & 3,495 & 6,313 \\ 0,70 & 0,507 & 0,877 & 1,238 & 1,595 & 1,948 & 3,183 & 3,265 & 3,384 & 6,304 \\ 0,75 & 0,408 & 0,707 & 0,999 & 1,288 & 1,576 & 3,168 & 3,221 & 3,299 & 6,297 \\ 0,80 & 0,316 & 0,548 & 0,774 & 0,999 & 1,223 & 3,158 & 3,189 & 3,236 & 6,291 \\ 0,85 & 0,230 & 0,398 & 0,563 & 0,727 & 0,891 & 3,150 & 3,167 & 3,192 & 6,287 \\ 0,90 & 0,149 & 0,258 & 0,365 & 0,471 & 0,577 & 3,145 & 3,152 & 3,163 & 6,285 \\ 0,95 & 0,072 & 0,126 & 0,178 & 0,229 & 0,281 & 3,142 & 3,144 & 3,147 & 6,284 \\ 1 & 0 & 0 & 0 & 0 & 0 & 3,142 & 3,142 & 3,142 & 6,283\end{array}$


Résonateur concentrique - Modes $\mathrm{TE}_{n m p}$ : valeurs de $k\left(R_{2}-R_{1}\right)$ satisfaisant l'équation $j_{n}\left(k R_{1}\right) v_{n}\left(k R_{2}\right)-j_{n}\left(k R_{2}\right) y_{n}\left(k R_{1}\right)=0$.

\begin{tabular}{|c|c|c|c|c|c|c|c|c|c|}
\hline \multirow[b]{2}{*}{$R_{1} / R_{2}$} & \multicolumn{9}{|c|}{$n, p$} \\
\hline & 1,1 & 2,1 & 3,1 & 4,1 & 5,1 & 1,2 & 2,2 & 3,2 & 1,3 \\
\hline 0 & 4,493 & 5,763 & 6,988 & 8,183 & 9,356 & 7,725 & 9,095 & 10,417 & 10,904 \\
\hline 0,05 & 4,272 & 5,475 & 6,639 & 7,773 & 8,888 & 7,356 & 8,641 & 9,896 & 10,404 \\
\hline 0,10 & 4,070 & 5,188 & 6,289 & 7,364 & 8,420 & 7,062 & 8,196 & 9,376 & 10,065 \\
\hline 0,15 & 3,895 & 4,907 & 5,940 & 6,955 & 7,952 & 6,851 & 7,790 & 8,863 & 9,860 \\
\hline 0,20 & 3,749 & 4,640 & 5,595 & 6,547 & 7,485 & 6,702 & 7,448 & 8,378 & 9,733 \\
\hline 0,25 & 3,629 & 4,393 & 5,257 & 6,140 & 7,017 & 6,597 & 7,178 & 7,948 & 9,649 \\
\hline 0,30 & 3,530 & 4,173 & 4,936 & 5,741 & 6,552 & 6,520 & 6,969 & 7,587 & 9,591 \\
\hline 0,35 & 3,448 & 3,980 & 4,638 & 5,355 & 6,094 & 6,463 & 6,808 & 7,296 & 9,549 \\
\hline 0,40 & 3,383 & 3,814 & 4,368 & 4,992 & 5,650 & 6,420 & 6,684 & 7,064 & 9,518 \\
\hline 0,45 & 3,330 & 3,674 & 4,130 & 4,658 & 5,229 & 6,386 & 6,588 & 6,881 & 9,495 \\
\hline 0,50 & 3,286 & 3,556 & 3,923 & 4,358 & 4,841 & 6,361 & 6,513 & 6,736 & 9,477 \\
\hline 0,55 & 3,251 & 3,458 & 3,745 & 4,095 & 4,491 & 6,341 & 6,454 & 6,621 & 9,464 \\
\hline 0,60 & 3,222 & 3,377 & 3,596 & 3,869 & 4,182 & 6,325 & 6,408 & 6,530 & 9,453 \\
\hline 0,65 & 3,199 & 3,312 & 3,473 & 3,677 & 3,917 & 6,313 & 6,372 & 6,459 & 9,445 \\
\hline 0,70 & 3,181 & 3,260 & 3,374 & 3,520 & 3,694 & 6,304 & 6,344 & 6,404 & 9,438 \\
\hline 0,75 & 3,168 & 3,219 & 3,295 & 3,393 & 3,512 & 6,296 & 6,323 & 6,362 & 9,434 \\
\hline 0,80 & 3,157 & 3,189 & 3,235 & 3,296 & 3,370 & 6,291 & 6,307 & 6,331 & 9,430 \\
\hline 0,85 & 3,150 & 3,167 & 3,192 & 3,224 & 3,265 & 6,287 & 6,296 & 6,308 & 9,428 \\
\hline 0,90 & 3,145 & 3,152 & 3,163 & 3,177 & 3,194 & 6,285 & 6,288 & 6,294 & 9,427 \\
\hline 0,95 & 3,142 & 3,144 & 3,147 & 3,150 & 3,154 & 6,284 & 6,284 & 6,286 & 9,426 \\
\hline 1 & 3,142 & 3,142 & 3,142 & 3,142 & 3,142 & 6,283 & 6,283 & 6,283 & 9,425 \\
\hline
\end{tabular}

ment remplie par un diélectrique, à faibles pertes, de permittivité $\varepsilon_{\mathrm{r}}$.

Il suffit de remplacer la célérité de la lumière dans le vide, $c$, par $c / \sqrt{\varepsilon_{\mathrm{r}} \text {. }}$

3. Conclusion. - L'utilisation des tables numériques présentées ici permet d'éviter le recours à un ordinateur pour la résolution numérique des équations (3), (4), (6) et (7). Il en résulte un gain de temps appréciable. De plus une représentation graphique, universelle, des résonances fait apparaître un classement des modes en deux familles pour lesquelles on peut calculer facilement les propriétés asymptotiques.

\section{Bibliographie}

[1] Waldron, R. A., Theory of guided electromagnetic waves

(Van Nostrand, London) 1970, p. 276.

[2] Dwight, H. B., J. Math. Phys. 27 (1948) 84-89. 